\title{
Reading habits for both words and numbers contribute to the SNARC effect
}

\author{
SAMUEL ShaKI \\ Ariel University Center of Samaria, Ariel, Israel \\ Martin H. Fischer \\ University of Dundee, Dundee, Scotland \\ AND \\ William M. Petrusic \\ Carleton University, Ottawa, Ontario, Canada
}

\begin{abstract}
This study compared the spatial representation of numbers in three groups of adults: Canadians, who read both English words and Arabic numbers from left to right; Palestinians, who read Arabic words and ArabicIndic numbers from right to left; and Israelis, who read Hebrew words from right to left but Arabic numbers from left to right. Canadians associated small numbers with left and large numbers with right space (the SNARC effect), Palestinians showed the reverse association, and Israelis had no reliable spatial association for numbers. These results suggest that reading habits for both words and numbers contribute to the spatial representation of numbers.
\end{abstract}

The spontaneous association between numbers and space has drawn much attention since its discovery (Dehaene, Bossini, \& Giraux, 1993). Well over 100 published experiments have shown that small-magnitude values are associated with the left side and larger values with the right side of space (for a recent meta-analysis, see Wood, Nuerk, Willmes, \& Fischer, 2008). The association is typically found by comparing the speed of rightand left-hand responses in a parity classification task. This so-called "spatial-numerical association of response codes" (SNARC) effect has been interpreted as reflecting a "spillover" of directional reading or writing habits. ${ }^{1}$ Readers in Western cultures progress through each line of text from left to right, and they also seem to place small numbers further on the left side of a "mental number line" than larger numbers when they enumerate objects or think about magnitudes. Supporting this explanation of the SNARC effect as a generalized habitual association, the spatial association for numbers was weaker in Iranians, who habitually read Arabic script from right to left but were only recently immersed into a left-to-right reading culture (Dehaene et al., 1993, Experiment 7). However, that study reported no data from Iranians in their native reading context, and thus no demonstration of the expected reversed association between numbers and space in right-to-left readers.

A field study by Zebian (2005) tried to address this point. She reported that monoliterate Arabic readers were faster to name the side of the larger number in a pair when it was on the left side of a display than when it was on the right, suggesting a reversed association between numbers and space consistent with their right-to-left reading habits. However, the verbal naming task is not sensitive to the SNARC effect (Keus \& Schwarz, 2005); this was evidenced by the absence of a normal effect in Zebian's English-speaking control group. Together, these results cast doubt on whether a reversed SNARC effect was indeed present in Zebian's (2005) study, or whether some other aspect of the task led to a spatial bias in these monoliterate Arabic readers.

Ito and Hatta (2004; see also Schwarz \& Keus, 2004) reported a vertical SNARC effect (small numbers associated with lower keys and larger numbers with upper keys) in Japanese readers. This association conflicts with the habitual top-to-bottom reading direction in Japanese and suggests that reading habit and SNARC effect might be independent. Recently, Hung, Hung, Tzeng, and Wu (2008) showed a horizontal mapping for Arabic number symbols, which their Chinese participants typically encountered in horizontally printed English text, and a vertical mapping for Chinese number symbols, which most often appeared in vertically printed Chinese text. Such flexibility in the same group of readers in the association of numbers with space resulting from both the number format and the associated reading context suggests that number concepts might not possess spatial associations in their own right. Instead, it seems possible that separate spatial associations become activated from the number itself and also from

S.Shaki, samuel_shaki@hotmail.com 
the associated reading context, and that their combination determines the resulting SNARC effect.

To test this possibility, we measured the SNARC effect with a parity task in three groups of participants with different combinations of spatial directional habits associated to the processing of words and numbers: Canadians, who habitually read and write both English words and Arabic numbers from left to right; Israelis, who habitually read and write Hebrew words from right to left but Arabic numbers from left to right; and Palestinians, who read and write both Arabic text and Arabic-Indic numbers (also called Eastern Arabic numerals) from right to left. We expected a normal left-to-right SNARC effect in the Canadians, a reversed right-to-left SNARC effect in the Palestinians, and a diluted SNARC effect in the Israelis because of their conflicting spatial associations for words and numbers.

\section{METHOD}

\section{Participants}

Twelve Canadian Carleton University students ( 7 female, 5 male, ages 18-24 years, 1 left-handed), 16 Israeli students from Ariel University Center of Samaria (12 female, 4 male, ages 19-23 years, 3 left-handed) and 11 Palestinians ( 2 female, 9 male, ages 19-25 years, 2 left-handed; mostly Islamic College students and some young noncollege students) took part in this study. The Palestinian participants read Arabic only and used Arabic-Indic numerals (where both words and numbers are written from right to left) and reported minimal exposure to any left-to-right written language. The Canadians and Israelis participated for course credit, but the Palestinians volunteered. They had normal or corrected-to-normal vision and participated in a single 30-min session.

\section{Stimuli and Design}

Either the Arabic digits 1, 2, 3, 4, 6, 7, 8, and 9 (for Canadians and Israelis) or the corresponding Arabic-Indic digits $1, r, r, \varepsilon, 7$, $\vee, \wedge$, and $\uparrow$ (for Palestinians) appeared in black Times New Roman font (size 30) on a white background at the center of the screen of a 17 -in. (43-cm, $800 \times 600$ pixel resolution) monitor. Responses were made on a standard keyboard, with all keys covered except "A" (for left-hand responses) and "L" (for right-hand responses).

Procedure. Participants were tested individually in a dimly lit room, seated approximately $50 \mathrm{~cm}$ from the center of the screen. They were instructed that on each trial a number would appear and they would have to indicate with a fast key response whether it was odd or even. Each trial ended with the participant's response, and the next trial started after 1,000 msec. All eight digits appeared randomly and equally often in each of two blocks with opposite response assignments (even-left key or even-right key), resulting in 160 trials in the Palestinian group and 128 trials in the Canadian and Israeli groups. ${ }^{2}$ Block order was counterbalanced across participants, and there was a short practice phase before data collection.

Analysis. Two Israeli participants had error rates $>30 \%$ and were excluded from further analyses; all other participants had $<15 \%$ errors. Correct reaction times (RTs) within 200-1,500 msec (amounting to $97 \%$ of data) were averaged for each digit and each hand. Mixed-factor ANOVAs evaluated effects of group (Canadian, Israeli, Palestinian), number magnitude (small: 1, 2, 3, 4; large: 6, 7, 8, 9), number parity (odd, even), and response key (left, right) on overall performance in terms of correct RTs and error percentages.

Next we calculated the RT difference (right minus left hand) for each digit and regressed it on digit magnitude as a predictor to determine the strength and direction of the SNARC effect for each participant (cf. Fias \& Fischer, 2005). Negative slope coefficients reflect the typical Western association between numbers and space. We also included digit parity $(-.5$ for even, +.5 for odd; cf. Andres, Davare, Pesenti, Olivier, \& Seron, 2004) as a predictor in a multiple regression.

\section{RESULTS}

\section{Overall Performance}

Average RT was $610 \mathrm{msec}(\mathrm{SE}=19 \mathrm{msec})$, with no between-group differences $[F(2,34)=0.95, p>.39]$. Group means were $574 \mathrm{msec}(\mathrm{SE}=32 \mathrm{msec}), 629 \mathrm{msec}$ $(\mathrm{SE}=30 \mathrm{msec})$, and $626 \mathrm{msec}(\mathrm{SE}=33 \mathrm{msec})$, for $\mathrm{Ca}-$ nadians, Israelis, and Palestinians, respectively. There was a main effect of parity $[F(1,34)=20.77, p<.001]$, indicating an "odd effect" (see Hines, 1990; Nuerk, Iversen, \& Willmes, 2004), with slower responses for odd than for even numbers (624 msec and $596 \mathrm{msec}$, respectively). The only other reliable effect was the predicted triple interaction between number magnitude, response key, and group $[F(2,34)=16.74, p<.001]$. It reflected the fact that, under the SNARC-compatible mapping of numbers to keys (small-left, large-right), there was a reliable 24msec RT benefit in Canadians $[t(11)=2.79, p<.02]$, a nonreliable $7-\mathrm{msec}$ RT penalty in Israelis $[t(13)=1.57$, $p>.14]$, and a highly reliable 33-msec RT penalty in Palestinians $[t(10)=6.41, p<.001]$ when compared with the opposite mapping.

Average error rate was $5.3 \%(\mathrm{SE}=0.9 \%)$, with no between-group differences $(F<1)$. Error rates replicated the odd effect, with $6.5 \%$ errors for odd and $4.0 \%$ errors for even numbers $[F(1,34)=9.04, p<.01]$. The triple interaction between digit magnitude, response key, and group was marginal in error rates $[F(2,34)=3.24, p=$ .051] but consistent with the RT pattern: Compared with the reverse mapping, under the SNARC-compatible mapping of numbers to keys (small-left, large-right), there was a $1.2 \%$ benefit in Canadians, a $0.9 \%$ benefit in Israelis, and a $4 \%$ penalty in Palestinians.

\section{Regression Slopes}

The results of the multiple regression analyses are displayed in Figure 1. For Canadians, the average slope coefficient for magnitude was $-10.81 \mathrm{msec} / \mathrm{unit}(S D=12.12$, range $=-27.03$ to 0.05 ), indicating a significant SNARC effect $[t(11)=3.09, p<.01]$, with small numbers associated to left responses and large numbers to right responses. The average slope coefficient for magnitude for the 14 Israelis was $1.26 \mathrm{msec} /$ unit $(S D=9.10$, range $=$ -8.22 to 14.08 ), reflecting the absence of a systematic association of numbers with space in this group $[t(13)=$ $0.66, p>.52]$. Finally, for Palestinians, the average slope coefficient for magnitude was $+12.56 \mathrm{msec} /$ unit $(S D=$ 8.19 , range $=4.43$ to 31.9 ), showing a significant reverse SNARC effect $[t(10)=5.09, p<.001]$, with small numbers associated to right responses and large numbers to left responses. The slope difference of $12 \mathrm{msec} /$ digit between Canadians and Israelis was significant $[t(24)=3.15, p<$ $.01]$, as was the $11-\mathrm{msec}$ difference between Israelis and Palestinians $[t(23)=3.69, p<.01]$.

Regression weights for parity were $-3.21,23.93$, and 31.84 for Canadian, Israeli, and Palestinian participants, 


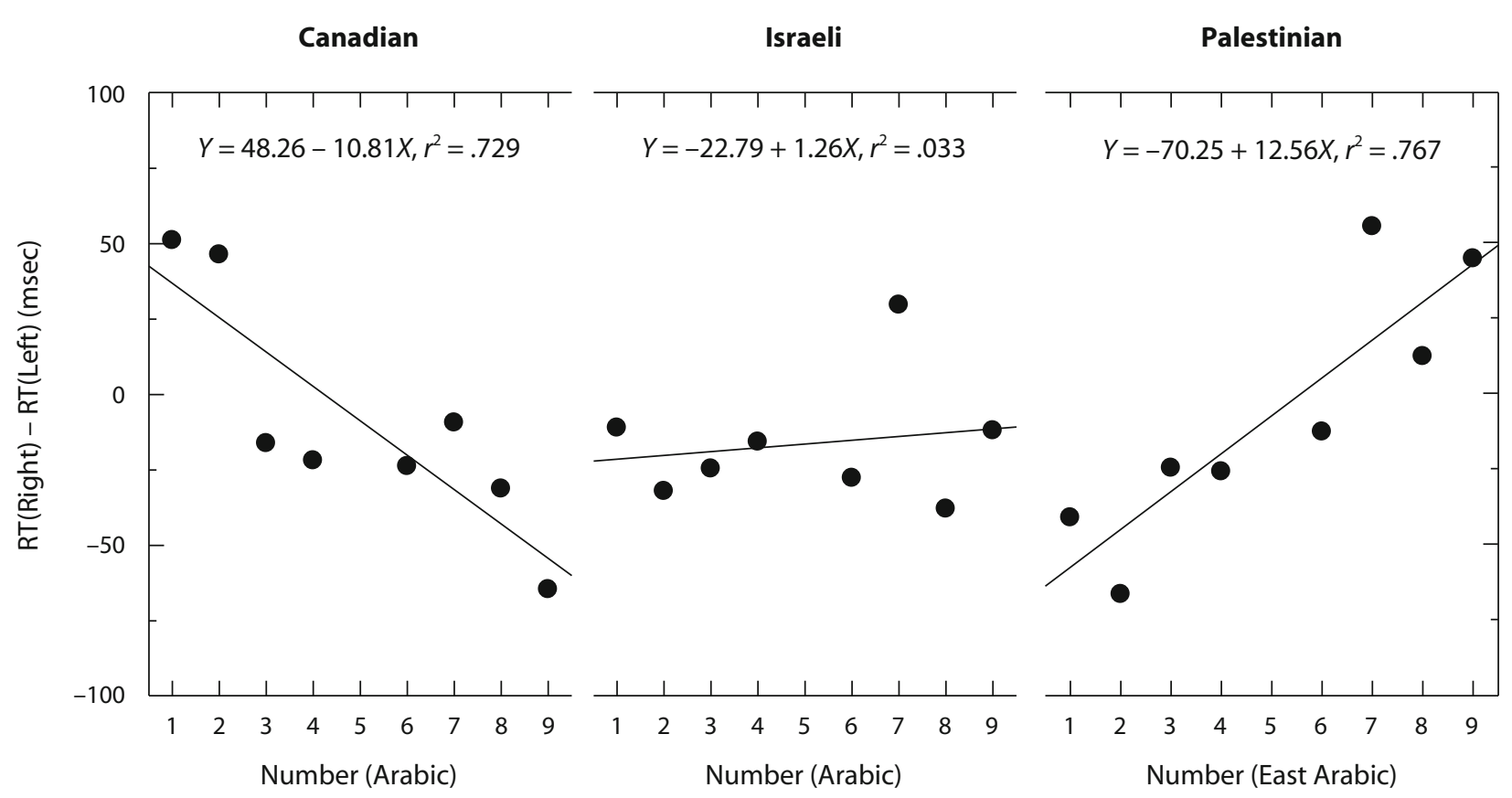

Figure 1. Mean SNARC effect [RT(right) - RT(left) in msec] as a function of digit presented in the parity task with Canadian, Israeli, and Palestinian participants. Direction of reading words and numbers for each group indicated by arrows.

respectively (all $p \mathrm{~s}>.08$ ). Thus, parity was not reliably linked to the response side, indicating the absence of a MARC effect (Nuerk et al., 2004) in all groups.

At the overall group level, all 11 Palestinians exhibited positive slopes, and 10 of the 12 Canadians had negative slopes, whereas 9 Israelis had positive slopes and $5 \mathrm{had}$ negative slopes. In neither of the Israeli groups were orderly SNARC effects evident. Thus, it is clear that the absence of SNARC, so strikingly evident in Figure 1, is not an artifact of averaging across the two subgroups. Confirming the conclusion that the SNARC effects changed with reading experience for both words and numbers, a between-group ANOVA on the slope coefficients was highly significant $[F(2,34)=18.14, p<.001]$, and Bonferroni-corrected post hoc tests found all pairwise between-group contrasts to be reliable $(p<.02)$. When RT was considered as covariate in an ANCOVA, it did not account for a significant amount of variance $[F(1,33)=$ $0.03, p=.863]$.

\section{DISCUSSION}

This study has two important results. First, by testing Palestinians in the standard parity task with Arabic-Indic digits, we demonstrated, for the first time, a clear association between small numbers and right space and between large numbers and left space. This observation constitutes a reversed SNARC effect when compared with the welldocumented association pattern in Western cultures.

Second, we compared three groups of participants with different combinations of spatial directional habits associated to the processing of words and numbers. This comparison revealed that the directional habits associated with both text and numbers contribute to the overall SNARC effect. Specifically, we showed that the SNARC effect is present when the reading directions for numbers and words are consistent (both from left to right, Canadian group; or both from right to left, Palestinian group), but it is absent when the two directions are inconsistent (in the Israeli group, words are read from right to left and numbers from left to right).

Of course, there are several other cultural differences among our groups, some of which might affect the mapping of numbers onto space: finger counting preferences (e.g., Fischer, 2008), for example, or general spatial heuristics that may be present before reading acquisition (e.g., Opfer \& Thompson, 2006; Tversky, Kugelmass, \& Winter, 1991). However, the Opfer and Thompson findings of directional preferences for enumeration in preschoolers may reflect factors other than general prelinguistic predispositions to spatial components in the mental representation of numbers. For example, children as young as 3 years old will have observed their parents reading and, indeed, pointing to words while reading. Also, 4- and 5-year-old children may encounter numerical displays in daycare centers and in kindergarten. A clear implication of this notion is that Israeli and Palestinian children will begin counting an array of objects from right to left, in contrast to children of parents living in a different culture who read from left to right.

Although the direction of reading is perfectly tied to the direction of SNARC for the horizontal dimension, this is not the case with the vertical dimension. For example, Schwarz and Keus (2004) demonstrate SNARC in the vertical dimension with small numbers at the bottom and large at the top. Surprisingly, Ito and Hatta (2004) also found vertical SNARC paralleling that of Schwarz and Keus, 
even though their participants read Japanese text in columns from top to bottom. On the other hand, Hung et al. (2008) did find a vertical SNARC effect corresponding to the direction of reading - that is, from top to bottom. Thus, it is clear that SNARC effects in the vertical dimension are not so closely entrained to the direction of reading.

In a recent study, we (Shaki \& Fischer, 2008) found that reading a short paragraph of text in either Hebrew or Russian systematically modulated SNARC in bilingual readers. Thus, activating one of several preexisting directional spatial habits led to differences in the mapping of numbers to space. That study complements the present work by showing that the default spatial mapping for numbers depends on recent activities. The present article documents the effects of the consistency between preexperimentally existing spatial mappings on the SNARC effect, whereas Shaki and Fischer (2008) reported effects of experimentally induced spatial mappings on the SNARC effect.

Our results support the hypothesis of a link between reading direction and SNARC effect, as was first inferred by Dehaene et al. (1993), but also suggest that, in addition, reading habits for numbers are an equally important factor. From this perspective, our use of the Arabic-Indic number stimuli was important in bringing about the reversed SNARC effect in Palestinians, because this surface format probably activated the habitual right-to-left processing strategy associated with such numbers (see also Hung et al., 2008). This spatial-numerical association was consistent with the other long-term spatial association of these participants, resulting from their directional reading habits. We think that this consistency enables the association between numbers and space to become significant.

\section{AUTHOR NOTE}

The work was supported in part by a Natural Sciences and Engineering Research Council of Canada Individual Discovery Grant to W.M.P. M.H.F. is a member of the Marie Curie Research and Training Network: Language and Brain (www.hull.ac.uk/RTN-LAB/). His work is funded by the European Commission (MRTN-CT-2004-512141 and ECRPRES-000-23-1388) as part of its Sixth Framework Program. Correspondence concerning this article should be addressed to S. Shaki, Department of Behavioral Sciences, Ariel University Center of Samaria, Ariel 44837, Israel (e-mail: samuel_shaki@hotmail.com).

\section{REFERENCES}

Andres, M., Davare, M., Pesenti, M., Olivier, E., \& Seron, X. (2004). Number magnitude and grip aperture interaction. NeuroReport, 15, 2773-2777.
Dehaene, S., Bossini, S., \& Giraux, P. (1993). The mental representation of parity and number magnitude. Journal of Experimental Psychology: General, 122, 371-396.

FiAs, W., \& FISCHER, M. H. (2005). Spatial representation of numbers. In J. I. D. Campbell (Ed.), Handbook of mathematical cognition (pp. 4354). New York: Psychology Press.

Fischer, M. H. (2008). Finger counting habits modulate spatialnumerical associations. Cortex, 44, 386-392.

Hines, T. M. (1990). An odd effect: Lengthened reaction times for judgments about odd digits. Memory \& Cognition, 18, 40-46.

Hung, Y.-H., Hung, D. L., Tzeng, O. J.-L., \& Wu, D. H. (2008). Flexible spatial mapping of different notations of numbers in Chinese readers. Cognition, 106, 1441-1450.

ITO, Y., \& HATTA, T. (2004). Spatial structure of quantitative representation of numbers: Evidence from the SNARC effect. Memory \& Cognition, 32, 662-673.

Keus, I. M., \& Schwarz, W. (2005). Searching for the locus of the SNARC effect: Evidence for a response-related origin. Memory \& Cognition, 33, 681-695.

Nuerk, H. C., Iversen, W., \& Willmes, K. (2004). Notational modulation of the SNARC and the MARC (linguistic markedness of response codes) effect. Quarterly Journal of Experimental Psychology, 57, 835-863.

Opfer, J. E., \& Thompson, C. A. (2006). Even early representations of numerical magnitude are spatially organized: Evidence from a directional magnitude bias in pre-reading preschoolers. In R. Sun \& N. Miyaki (Eds.), Proceedings of the 28th Annual Conference of the Cognitive Science Society (pp. 639-644). Mahwah, NJ: Erlbaum.

Schwarz, W., \& Keus, I. (2004). Moving the eyes along the mental number line: Comparing SNARC effects with manual and saccadic responses. Perception \& Psychophysics, 66, 651-664.

ShAKI, S., \& Fischer, M. H. (2008). Reading space into numbersA cross-linguistic comparison of the SNARC effect. Cognition, 108, 590-599.

Tversky, B., Kugelmass, S., \& Winter, A. (1991). Cross-cultural and developmental trends in graphic productions. Cognitive Psychology, 23, 515-557.

Wood, G., Nuerk, H. C., Willmes, K., \& Fischer, M. H. (2008). On the cognitive link between space and number: A meta-analysis of the SNARC effect. Psychology Science Quarterly, 50, 489-525.

ZeBIAN, S. (2005). Linkages between number concepts, spatial thinking, and directionality of writing: The SNARC effect and the reverse SNARC effect in English and Arabic monoliterates, biliterates, and illiterate Arabic speakers. Journal of Cognition \& Culture, 5, 166-190.

\section{NOTES}

1. In the remainder of this article we use the term reading habits to refer to both reading and writing, because the directions of reading and writing are perfectly correlated.

2. The slightly smaller number of trials for Canadians and Israelis was due to different time constraints during data collection.

(Manuscript received January 7, 2008; revision accepted for publication October 3, 2008.) 